\title{
Clinical and Translational Oncology: a national high-impact journal and an objective international expansion
}

\author{
The Editorial Board
}

$\mathrm{T}$ oday it is relatively easy to gauge the relevance of a journal and its international impact and visibility. According to its international impact factor (IF) a journal can be assigned as one of the leading journals in its speciality, as an outstanding or moderate journal or as not influential at all. But everything is relative in this world of strong competition, and the influence of a scientific journal can be also be determined by less international and more local parameters.

What if we determined the influence of a journal in its own country? This is also happening with general journals and magazines. The very well known global journals -no need to name them here- indeed have a worldwide visibility and a worldwide impact. However, there is no doubt that national journals may have even higher visibility and influence in their own environment. They are what most people read and consider in their day-to-day life. The same could apply to scientific journals; some local journals may have a higher national impact than leading journals due to their better accessibility or distribution.

In Spain there are not many specialised journals dedicated to publishing cancer research. Among these, Clinical and Translational Oncology is the most relevant, exclusively dedicated to this field. More than 2500 cancer professionals receive this journal every month directly to their home or place of work. This is the bulk of Spanish oncologists. And many more are exposed to the journal indirectly through the libraries of their hospitals, universities and research centres; therefore the journal reaches all the relevant laboratories, university departments and clinical oncology settings.

Clinical and Translational Oncology is devoted to fostering communication among all Spanish professionals dedicated to dealing with all aspects of cancer, from education and research to clinical management and treatment. The programmes to fulfil the highest demands of continuous medical education are highly appreciated among all members of the Spanish cancer societies integrated in the Federation of Spanish Cancer Societies (FESEO). These papers are heavily cited in journals included in the Science Citation Index (SCI), an objective proof of their value and recognition.

\begin{tabular}{ll} 
Top $\mathbf{5}$ Asia-Pacific & \\
\hline - China & $5 \%$ \\
- Iran & $4 \%$ \\
- South Korea & $3 \%$ \\
- Turkey & $3 \%$ \\
- Taiwan & $2 \%$
\end{tabular}


In addition, Clinical and Translational Oncology is an extraordinary vehicle to help in reporting original research -both basic and clinical- performed in those laboratories and hospitals that are starting to get involved in these activities, as well as those already well established and known.

Thus, there is no doubt that Clinical and Translational Oncology has an enormous impact at the local level in Spain, and determined by this parameter is the most relevant journal devoted to all aspects of cancer research and treatment in our country.

Since January 2005, Clinical and Translational Oncology has been indexed in Medline and its visibility has been boosted also at the international level in a progressive manner thanks to the strategic collaboration with Springer for international dissemination. In addition, the possibility of accessing the published manuscripts electronically through the web has further increased its international impact. This will soon be followed and measured through the SCI year report, starting this year. Therefore, in addition to its highly recognised national impact, our journal is starting to become a player in the international arena.
Today we can celebrate that our journal has emerged very strongly at this international level, since we had over 18,500 full-article electronic downloads worldwide in 2008. And what it is more important, the mean value from the last 4 months indicates that we should expect over 30,000 fullarticle downloads in 2009. The distribution of these downloads from January to June 2008 reflects an outstanding achievement (see graphic) since the downloads were mostly in North America (41\%), Asia-Pacific (26\%) and Europe $(22 \%)$. Spain has only a $4 \%$ share of the total, which alone indicates that the journal is not only an outstanding local journal but has become a truly international one.

It is indeed a good starting point for our international launch, which we believe will be further boosted when we are included in the SCI year report.

Therefore, Clinical and Translational Oncology is the leading cancer journal in Spain with a high real impact among cancer professionals, and it is becoming relevant in the international context.

The Editorial Board wants to thank all members of FESEO for their continued support that encourages us to continue on this ongoing progressive path. 